\title{
Lid abscess: An unusual presentation of melioidosis
}

\section{Bhagwati Wadwekar ${ }^{1}$, Rohan Suresh Ninan ${ }^{1}$, Sandhya Bhat ${ }^{2}$, Sheela Devi², Ramaya SR ${ }^{2}$, and Reba}

\author{
Kanungo ${ }^{2}$
}

1. Department of Ophthalmology, Pondicherry Institute of Medical Sciences, Puducherry, India

2. Department of Microbiology, Pondicherry Institute of Medical Sciences, Puducherry, India

\section{CASE STUDY}

Please cite this paper as: Wadwekar B, Ninan SR, Bhat S, Devi S, Ramya SR, Kanungo R. Lid abscess: An unusal presentation of melioidosis. AMJ 2018;11(6):322-325. https://doi.org/10.21767/AMJ.2017.2809

Corresponding Author:

Bhagwati Wadwekar

Department of Ophthalmology

Pondicherry Institute of Medical Sciences, Kalapet,

Puducherry 605014, India

Email: bwadwekar@yahoo.com

\section{ABSTRACT}

Melioidosis caused by Burkholderia pseudomallei (B. pseudomallei), is an emerging infection in India. Clinical manifestation of melioidosis is variable ranging from localized benign infection to fulminant septicemia. Ocular presentation of melioidosis is rare. However, cases of endophthalmitis, keratitis and orbital infections have been reported. We report the isolation of $B$. pseudomallei in a 40 year old male, presenting with fever and lid abscess. The patient was treated with meropenem during the acute phase and switched to trimethoprim/sulfamethoxazole for eradication phase.

\section{Key Words}

Burkholderia, endophthalmitis, keratitis

\section{Implications for Practice:}

\section{What is known about this subject?}

The clinical spectrum of melioidosis varies from localised benign infection to fulminant sepsis. Ocular presentation is rare. Endophthalmitis, keratitis and orbital infections have been previously reported.

\section{What new information is offered in this case study?}

There are few case reports that show B. pseudomallei can cause orbital cellulitis. It's presentation as lid abscess in isolation without orbital cellulitis is not described.

3. What are the implications for research, policy, or practice?

Our case report highlights that in endemic areas of melioidosis, lid abscess could be one of the clinical manifestations.

\section{Background}

Melioidosis is a common tropical infection in South East Asia. ${ }^{1-5}$ It is endemic in Australia, Thailand, and several parts of the South Asia regions like India and Srilanka. In India melioidosis has been reported sporadically from several of the country. Large number of cases has been reported from South Western regions namely Manipal, Mangalore and surrounding regions in Karnataka. ${ }^{6-8}$ In the absence of laboratory support this disease may be underdiagnosed and hence few cases are reported in the literature. Ocular presentations of melioidosis are rare. However cases of endophthalmitis, keratitis and orbital infections have been reported in the literature. We describe an interesting case of lid abscess caused by $B$. pseudomallei admitted to a tertiary care hospital.

\section{Case details}

A 40 year old male resident of adjoining rural Tamil Nadu presented with a history of intermittent fever for 12 days, external swelling on the tip of his nose and pain over the left side of the face for 10 days.

He was a known diabetic on treatment with oral hypoglycaemic drugs. He had been admitted to the local government hospital and was diagnosed with left nasal vestibulitis for which an incision and drainage was done. Subsequently he developed profound hyponatremia (Table 1) and was referred to the present hospital. 
On examination the patient was conscious and oriented, with a body temperature of $98.6^{\circ} \mathrm{F}$, pulse rate was 72 /minutes and blood pressure $190 / 60 \mathrm{~mm} \mathrm{Hg}$. Other than hypertension, no abnormality was detected on systemic examination. Swelling and warmth were felt on the tip of the nose extending into left ala. This area was tender. The complete blood picture including total leukocyte counts (TLC) and erythrocyte sedimentation rate (ESR) was essentially normal (Table 1 ). Biochemistry panel revealed raised blood sugar levels, blood urea levels and serum creatinine. The values have been summarized in Table 2 .

A working diagnosis of left nasal vestibulitis, along with hyponatremia, acute kidney injury and uncontrolled diabetes mellitus was made. The patient was started on empiric treatment of intravenous Metronidazole $500 \mathrm{mg}$ thrice daily and intravenous Cefoperazone-sulbactam $1.5 \mathrm{gm}$ twice a day. However the patient continued to have fever. After seven days of admission he developed swelling of the left eye. Ocular examination showed a swelling over the medial third of the left lower lid (Figure 1). It was fluctuant, tender and inflamed suggesting collection of pus. The remainder of the anterior segment and fundus examinations were normal. A diagnosis of left side lower lid abscess was made. Computerized tomography (CT) of the orbit and paranasal sinus showed soft tissue swelling in the left nasal and pre-septal medial left lower lid. Right sided mild sinusitis and a nasal polyp were also noted.

The lid abscess was drained and pus was sent for culture and sensitivity. Direct gram stain from pus revealed gram negative bacilli, with a few bacilli showing safety pin appearance. Overnight incubation on blood agar showed dry wrinkled cream coloured colonies and on MacConkey agar pink coloured dry colonies with a metallic sheen (Figure 2A and B). The isolate was identified as $B$. pseudomallei based on standard biochemical tests. ${ }^{9}$ Antibiotic susceptibility testing was done by the Kirby Bauer disc diffusion method and the isolate was found to be sensitive to ceftazidime, piperacillin tazobactam, imipenem, meropenem and trimethoprim/sulfamethoxazole. Simultaneous blood culture also grew B. pseudomallei. Both the isolates were confirmed by Vitek2 compact. Computerized tomography of chest and abdomen was done and did not reveal any other foci of infection.

The patient was started on an acute phase treatment of meropenem 1gm intravenous twice daily for two weeks and then switched over to eradication phase with trimethoprim/sulfamethoxazole (80mg/400mg), three tablets every 12 hours. The patient was discharged with the advice to continue the eradication phase of trimethoprim/sulfamethoxazole. After 12 weeks of eradication phase blood culture did not yield any organisms.

\section{Discussion}

Lid abscess caused by B. pseudomallei has not been described so far. Our case also had systemic infection in addition to lid abscess. Although melioidosis is endemic in India, its ocular presentations like endophthalmitis, keratitis, orbital cellulitis and lid abscess are uncommon. ${ }^{1-5}$

Lid abscess is generally caused by trauma, local spread of infection from surrounding adnexa like internal hordeolum, sinusitis, and endogenous spread through blood.8 Staphylococcus aureus and Group A $\beta$-haemolytic Streptococci are the most common organisms associated with these manifestations. ${ }^{10,11}$ If untreated it can lead to fistula formation and orbital cellulitis with loss of vision. In the present case the patient may have started with vestibulitis due $B$. pseudomallei infection which could have spread to the lid causing lid abscess. Although the patient was initially admitted in a local government hospital where incision and drainage was done for the vestibulitis, the pus was not sent for microbiological investigations and so the diagnosis of melioidosis was missed.

Ocular involvement is rare in melioidosis. There are few case reports that describe orbital cellulitis caused by this organism. ${ }^{2-5}$ Saonanon $P$ reported orbital cellulitis with subperiosteal abscess caused by $B$. pseudomallei leading to orbital apex syndrome. ${ }^{5}$ Chen $\mathrm{KJ}$ et al. reported a case of endogenous endophthalmitis caused by $B$. pseudomallei that was treated with systemic and intravitreal. ${ }^{1}$ Our case is unique because patient had lid abscess in isolation without any involvement of orbit (Figure 1). A previous review by Detporntewan $P$ reported four cases of orbital cellulitis caused by melioidosis. One of them had lid involvement in the form of preseptal abscess. This case in fact presented with orbital cellulitis, and lid abscess was revealed only by CT orbit. ${ }^{3,4}$ This fact that melioidosis can present as solitary swelling over lid underscores the importance of sending microbiological assay for each and every such cases.

Our patient was farmer by occupation and though he was not a previously diagnosed case of diabetes mellitus, he had high fasting and post prandial sugar levels at admission. Diabetes mellitus is the most common underlying disease associated with melioidosis in most studies. ${ }^{12}$ Other predisposing factors identified are chronic renal disease and chronic alcoholic liver disease. Results of regression analysis in case control study by Yupin Suputtamongkol et al. 
confirmed diabetes mellitus, pre-existing renal diseases, thalassemia, and occupational exposure to be significant risk factors for melioidosis. In areas of endemic melioidosis, diabetics were at higher risk of developing melioidosis, especially septicemic disease. Significant interaction between diabetes mellitus and occupation was found in their study. They found occupation involving high exposure to soil as a risk factor for melioidosis and hence being a farmer might have put our patient at a higher risk of acquiring this infection. ${ }^{12}$

International consensus recommendations for the treatment and prophylaxis of melioidosis and glanders were developed by US Public Health Emergency Medical Countermeasures an expert group in $2010 .^{13}$ Treatment is usually done in two phases: acute phase and eradication phase. Acute phase treatment is to prevent severe sepsis. For patients without complications (i.e. without neurologic, prostatic, bone, or joint involvement), Ceftazidime $50 \mathrm{mg} / \mathrm{kg}$ (up to $2 \mathrm{~g}$ ) intravenous every eight hours, or $6 \mathrm{~g} /$ day by continuous infusion after a $2 \mathrm{~g}$ bolus is recommended for 10 14 days. However more than four weeks therapy may be necessary in severe infection such as septic shock, deepseated organ abscess, extensive lung disease, osteomyelitis, septic arthritis and neurological melioidosis. Trimethoprim/sulfamethoxazole can be added in patients with severe infection involving the brain, prostate or other privileged sites. Switching to meropenem is indicated if patient condition worsens while receiving ceftazidime, e.g., organ failure, development of a new focus of infection during treatment, or if repeat blood cultures remain positive. With neuromelioidosis, persistent bacteraemia or in the intensive care unit Meropenem $25 \mathrm{mg} / \mathrm{kg}$ (up to $1 \mathrm{~g}$ ) intravenous every eight hours for 10 to 14 days (four weeks in severe disease) should be used. For the eradication phase continuation of therapy in the form of oral drugs to prevent relapse is recommended for duration of minimum of 12 weeks. If the organism is resistant to trimethoprim/sulfamethoxazole or the patient is intolerant, alternative is amoxicillin/clavulanic acid. Supportive care with attention to underlying conditions is needed. ${ }^{13,14}$

Drainage of abscesses is recommended in addition to antibiotic treatment. Culture of the material is mandatory to confirm the diagnosis, as the treatment is very specific in two phases. Mild and localised infections can be cured by only oral agents. This patient was started on an acute phase treatment of meropenem $1 \mathrm{~g}$ intravenous twice daily for two weeks and then switched to eradication phase with trimethoprim/sulfamethoxazole (80mg/400mg), three tablets every 12 hours. Meropenem dosage was adjusted for abnormal renal parameters (Table 2) and given at a lower dose.

\section{Conclusion}

In conclusion lid abscess is a rare presentation of melioidosis. Surgical drainage of abscess is important part of management. Standard antibiotic treatment (acute phase \& maintenance phase) is advocated. B. pseudomallei may be responsible for larger number of human ocular infection than is presently known. As there is the possibility of a fulminant course and high mortality ophthalmologists should be alert to this diagnosis and subject the drained pus or tissue to a microbiology laboratory for confirmation to avoid delay in treatment and prevent complications.

\section{References}

1. Chen KJ, Sun $\mathrm{MH}$, $\mathrm{Hou} \mathrm{CH}$, et al. Burkholderia pseudomallei endophthalmitis. J Clin Microbiol. 2007;45(12):4073-4.

2. Shawarinin J, Bakiah S, Zunaina E, et al. Orbital abscessa rare presentation of melioidosis. Malays J Med Sci. 2007;14:244.

3. Detporntewan P, Suankratay C. Orbital infection caused by Burkholderia pseudomallei: A mini-review. J Infect Dis Antimicrob Agents. 2011;29(2):99-103.

4. Wong PK, Ng PH. Melioidosis presenting with orbital cellulitis. Singapore Med J. 1996;37(2):220-1.

5. Saonanon $P$, Tirakunwichcha $S$, Chierakul W. Case report of orbital cellulitis and necrotizing fasciitis from melioidosis. Ophthal Plast Reconstr Surg. 2013;29(3):814.

6. Vidyalakshmi K, Lipika S, Vishal S, et al. Emerging clinicoepidemiological trends in melioidosis: analysis of 95 cases from western coastal India. Int J Infect Dis. 2012;16(7):491-7.

7. Saravu K, Vishwanath S, Kumar RS, et al. Melioidosis-a case series from south India. Trans R Soc Trop Med Hyg. 2008;120:18-20.

8. Gopalakrishnan R, Sureshkumar D, Thirunarayan MA, et al. Melioidosis: an emerging infection in India. J Assoc Physicians India. 2013;61(9):24-6.

9. Winn WC. Koneman's color atlas and textbook of diagnostic microbiology. Koneman EW, editor. Lippincott williams \& wilkins; 2006.

10. Casady DR, Zobal-Ratner JL, Meyer DR. Eyelid abscess as a presenting sign of occult sinusitis. Ophthal Plast Reconstr Surg. 2005;21(5):368-70.

11. Brook I. Microbiology of polymicrobial abscesses and implications for therapy. J Antimicrob Chemother. 2002;50(6):805-10. 
12. Suputtamongkol Y, Chaowagul W, Chetchotisakd P, et al. Risk factors for melioidosis and bacteremic melioidosis. Clin Infect Dis. 1999;29(2):408-13.

13. Lipsitz R, Garges S, Aurigemma R, et al. Workshop on treatment of and postexposure prophylaxis for Burkholderia pseudomallei and B. mallei Infection, 2010. Emerg Infect Dis. 2012;18(12):2.

14. Dance D. Treatment and prophylaxis of melioidosis. Int J Antimicrob Agents. 2014;43(4):310-8.

\section{ACKNOWLEDGEMENTS}

Dr Anusha Rohit, Head of Microbiology, Madras Medical Mission for confirming the identity of the isolate.

\section{PEER REVIEW}

Not commissioned. Externally peer reviewed.

\section{CONFLICTS OF INTEREST}

The authors declare that they have no competing interests.

\section{FUNDING}

None

\section{PATIENT CONSENT}

The authors, Wadwekar B, Ninan R S, Bhat S, Devi S, Ramaya $S$, Kanungo $R$, declare that:

1. They have obtained written, informed consent for the publication of the details relating to the patient(s) in this report.

2. All possible steps have been taken to safeguard the identity of the patient(s).

3. This submission is compliant with the requirements of Institute ethics committee.

Figure 1: Lid abscess involving medial third of lower lid
Figure 2: (A) Growth on blood agar and (B) Macconkey agar

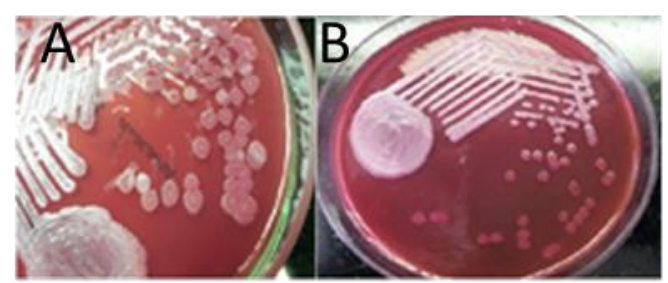

Table 1: Complete blood picture parameters

\begin{tabular}{|l|l|}
\hline Parameters & Values \\
\hline Total Leukocyte Count & $6.3 \times 10^{9} / \mathrm{cu} \mathrm{mm}$ \\
\hline Neutrophils & $72 \%$ \\
\hline Lymphocytes & $21 \%$ \\
\hline Basophils & $4 \%$ \\
\hline Eosinophils & $3 \%$ \\
\hline Erythrocyte Sedimentation Rate & $7 \mathrm{~mm}$ per hour \\
\hline Total Red Blood Cells & $4.2 \times 10^{12} / \mathrm{L}$ \\
\hline Packed Cell Volume & $33 \%$ \\
\hline Platelets & $360 \times 10^{9} / \mathrm{microliter}$ \\
\hline
\end{tabular}

Table 2: Blood Biochemistry parameters

\begin{tabular}{|l|l|}
\hline Parameters & Values \\
\hline Haemoglobin & $7.39 \mathrm{mmol} / \mathrm{L}$ \\
\hline Fasting Blood Sugar & $7.49 \mathrm{mmol} / \mathrm{L}$ \\
\hline Post Prandial Blood Sugar & $27.1 \mathrm{mmol} / \mathrm{L}$ \\
\hline Serum Urea & $50 \mathrm{mmol} / \mathrm{L}$ \\
\hline Serum Creatinine & $353.68 \mathrm{micromol} / \mathrm{L}$ \\
\hline Serum Sodium & $122 \mathrm{mmol} / \mathrm{L}$ \\
\hline Serum Pottasium & $3.1 \mathrm{mmol} / \mathrm{L}$ \\
\hline
\end{tabular}

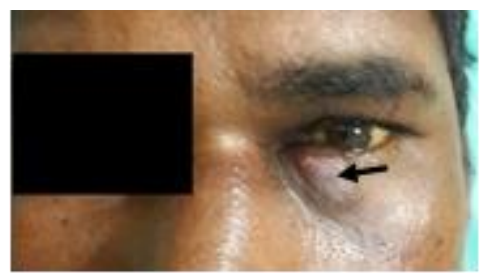

